\title{
CONTINUOUS TIME MEAN-VARIANCE PORTFOLIO OPTIMIZATION THROUGH THE MEAN FIELD APPROACH
}

\author{
MARKus Fischer $^{1}$ AND GiUlia Livieri ${ }^{2}$
}

\begin{abstract}
A simple mean-variance portfolio optimization problem in continuous time is solved using the mean field approach. In this approach, the original optimal control problem, which is time inconsistent, is viewed as the McKean-Vlasov limit of a family of controlled many-component weakly interacting systems. The prelimit problems are solved by dynamic programming, and the solution to the original problem is obtained by passage to the limit.
\end{abstract}

Mathematics Subject Classification. 91G10, 49L20, 82C22, 60H10.

Received January 30, 2014. Revised April 2, 2015. Accepted December 17, 2015.

\section{INTRODUCTION}

In this paper, we study and solve a mean-variance portfolio optimization problem in continuous time using the mean field approach introduced in the context of discrete time mean-variance problems by [2].

The problem of mean-variance portfolio optimization goes back, at least, to [11], who considered a single period (one time step) model. The model allows investors to be risk averse. Investment decisions are therefore made following two different objectives: to maximize expected return and to minimize financial risk. The two conflicting objectives can be combined; decisions are made so as to maximize a difference between expectation and variance of the random quantity representing wealth at terminal time. In a multi-period framework (discrete or continuous time models), this kind of optimization problem is time inconsistent in the sense that an investment strategy that is optimal over the entire time interval need not be optimal over subintervals. As a consequence, Bellman's Principle of Dynamic Programming does not hold for the underlying control problem.

Various approaches to multi-period mean-variance portfolio optimization have been explored; here, we mention four different approaches from the mathematically oriented literature.

The first approach, due to [10] in a discrete time setting, works by embedding the time inconsistent meanvariance optimization problem into a one-parameter family of standard time-consistent optimal control problems. By solving these problems for each value of the parameter and then choosing the right parameter value according to a compatibility constraint, one can solve the original problem. This technique is extended to continuous time models in [14].

\footnotetext{
Keywords and phrases. Portfolio optimization, mean-variance criterion, optimal control, time inconsistency, dynamic programming, McKean-Vlasov limit, law of large numbers.

1 Department of Mathematics, University of Padua, via Trieste 63, 35121 Padova, Italy. fischer@math.unipd.it

2 Scuola Normale Superiore di Pisa, Piazza dei Cavalieri 7, 56126 Pisa, Italy. giulia.livieri@sns.it
} 
A different approach is based on a game theoretic interpretation of time inconsistent optimal control problems. Time inconsistency is interpreted in terms of changes in agents' preferences, and an optimal control problem is viewed as a game where the players are the future incarnations of their own preferences. Solutions of the optimization problem are then defined in terms of sub-game perfect Nash equilibria; see [4] and the references therein. The framework is used in [5] to solve a continuous time mean-variance portfolio optimization problem with risk-aversion parameter that may depend on current wealth.

A third approach starts from the observation that dynamic mean-variance optimization problems can be seen as stochastic optimal control problems of McKean-Vlasov or mean field type (cf. [8]). For this class of control problems, the coefficients of the costs and dynamics may depend on the law of the controlled state process. As a consequence, the cost functional may be non-linear with respect to the joint law of the state and control process. In the context of mean-variance optimization, the coefficients of the controlled dynamics have standard form, while the cost (or gain) functional is a quadratic polynomial of the expected value of the state process; it is therefore non-linear as a functional of the law of the state process. The connection between mean-variance optimization and control of McKean-Vlasov type has been exploited in [1]. There, the authors derive a version of the stochastic Pontryagin maximum principle for continuous time optimal control problems of McKean-Vlasov type. The maximum principle is then used to obtain the optimal control for the mean-variance problem solved in [14]. The Pontryagin maximum principle for control problems of McKean-Vlasov type has also been studied in [6], where a necessary condition for optimality is derived provided that the non-linear dependence on the law of the state process can be expressed through a function of its expected value; in [7], the authors establish a necessary as well as a sufficient condition for optimality for systems with general dependence on the law of the state process, using a particular notion of differentiability for functions defined on a space of probability measures.

The work by [2], who treat the mean-variance portfolio optimization problem in discrete time, also builds on the connection with McKean-Vlasov control. Those authors, in order to circumvent the difficulties arising from time inconsistency, interpret the original control problem as the McKean-Vlasov limit of a family of controlled weakly interacting systems where interaction through the empirical measure appears only in the gain functional, while each component ("market clone") follows the same dynamics as that of the original controlled system. The approximate $K$-component control problem has the portfolio mean and portfolio variance replaced with their empirical counterparts, and dynamic programming can be used to determine the value function and optimal strategy. The original optimization problem is then solved by letting the number of components $K$ tend to infinity. The asymptotic connection between systems with measure-dependent coefficients and many-component (or many-particle) weakly interacting systems lies at the heart of the McKean-Vlasov theory, and it is well established in the case of uncontrolled systems; see, for instance, [12] or [13].

The Pontryagin principle approach mentioned above works directly with the limit model, that is, with the optimal control problem of McKean-Vlasov type. The law of the controlled state process, in particular, appears in both the forward and the adjoint equation. This approach is most useful when the convexity assumptions on the costs and the Hamiltonian required by the sufficient optimality condition are in force. This is the case for the problem at hand, which has a linear-quadratic structure if one includes the mean of the controlled state process. Still, for general convex models, solving the coupled non-linear system of forward and backward equation is difficult. The mean field approach in the spirit of [2], on the other hand, is based on the McKean-Vlasov limit and the standard theory of dynamic programming. No particular convexity structure is needed. The difficulty lies in finding sufficiently explicit solutions for the prelimit problems. The optimal prelimit strategies, if given in feedback form, should also be regular enough so that the passage to the McKean-Vlasov limit can be carried out.

Optimal control problems of McKean-Vlasov type are related to - but different from - what is known as mean field games. Mean field games are limit models for symmetric $N$-player games with interaction of mean field type. The optimality criterion for the prelimit models (i.e., the $N$-player games) is that of a Nash equilibrium. In the mean field limit, one representative player is left, while the behavior of all the other players is represented by a flow of probability measures on state space. For any given flow of measures, one has to solve an optimal 
control problem, the optimization problem for the representative player. A solution of the mean field game then consists in finding a flow of measures $\mathfrak{m}$ such that the flow of marginal distributions for the optimally controlled state process of the representative player given $\mathfrak{m}$ coincides with $\mathfrak{m}$. See again [8] for references and a comparison with optimal control problems of McKean-Vlasov type. Both types of problems have been solved rather explicitly for a class of linear-quadratic models in [3]; see, in particular, the comparison in Section 6 therein.

The aim of this paper is to show that the particle limit approach as introduced by [2] in discrete time can be successfully applied to continuous time models as well. We consider one of the simplest situations, namely a market model with exactly one risky asset and one risk-free asset. The risk-free asset is assumed to be constant, which corresponds to zero interest rate, while the evolution of the price of the risky asset is modeled as an arithmetic Brownian motion (Bachelier dynamics). The case of a geometric Brownian motion (Merton-BlackScholes dynamics) can be handled by re-interpreting the control processes: instead of controlling the (possibly negative) number of assets, control is exerted in terms of the market value of the assets. In the situation considered here, we obtain an explicit expression for the optimal investment strategy in feedback form. The result in itself is, of course, not new. It is essentially a special case of the solution obtained by [14] or [1]; $c f$. Remark 4.4 in Section 4 below. The result could also be derived by time discretization from the solutions of the discrete-time problems studied in [2]. Here, however, we rely on dynamic programming in continuous time. For the passage to the McKean-Vlasov limit, we use a coupling argument in the spirit of [13]. The contribution of the present paper therefore lies in the fact that we combine standard tools to solve a non-standard optimization problem, where the methods are suggested by the particle limit interpretation of the problem.

The rest of this paper is organized as follows. In Section 2, we formulate the mean-variance optimization problem and the corresponding finite horizon optimal control problem. In Section 3, we introduce auxiliary $K$-component (" $K$-clone") optimal control problems. Those problems, which are of linear-quadratic type, are solved explicitly using dynamic programming. In Section 4, we pass to the limit as the number of components (or "clones" or particles) $K$ tends to infinity. We obtain a limit feedback strategy and a limit value function, which are shown to yield the solution to the original optimization problem.

\section{ORiginAl OPTIMIZATION PROBLEM}

We consider a financial market consisting of two assets, one risky (for instance, a stock) and one non-risky (a bond). For simplicity, we assume that the interest rate for the bond is equal to zero, the bond price therefore constant and, without loss of generality, equal to one. The price of the risky asset, which we denote by $S$, is assumed to follow an arithmetic Brownian motion (or Brownian motion with drift). The process $S$ thus satisfies the equation

$$
\mathrm{d} S(t)=\mu \mathrm{d} t+\sigma \mathrm{d} W(t)
$$

with deterministic initial condition $S(0)=s_{0}>0$. In equation (2.1), $\mu>0$ is the average rate of return, $\sigma>0$ the volatility, and $(W(t))_{t \geq 0}$ a standard one-dimensional Wiener process defined on a filtered probability space $\left(\Omega_{\circ}, \mathcal{F}^{\circ},\left(\mathcal{F}_{t}^{\circ}\right), \mathbb{P}_{\circ}\right)$ satisfying the usual hypotheses.

Taking the point of view of a small investor, let $u(t)$ denote the (not necessarily integer) number of shares of the risky asset he or she holds at any given time $t$, and let $X(t)$ be the value of the corresponding self-financing portfolio, which consists of the risky and the risk-free asset. The process $X$ then evolves according to

$$
\mathrm{d} X(t)=\mu u(t) \mathrm{d} t+\sigma u(t) \mathrm{d} W(t)
$$

with deterministic initial condition $X(0)=x_{0}$, where $x_{0}>0$ is the initial capital. There is an implicit constraint on the investment strategy $u$ due to the stochastic environment of the problem, namely, $u$ is allowed to depend on the evolution of the random processes only up to the current time. The strategy $u$ must therefore be nonanticipative, that is, $u$ has to be $\left(\mathcal{F}_{t}^{\circ}\right)$-adapted. Below, we assume that $u$ belongs to $H_{T}^{2}\left(\left(\mathcal{F}_{t}^{\circ}\right)\right)$, the space of all real-valued $\left(\mathcal{F}_{t}^{\circ}\right)$-progressively measurable processes $v$ such that $\mathbb{E}\left(\int_{0}^{T}|v(t)|^{2} \mathrm{~d} t\right)<\infty$, where $T>0$ denotes the 
finite time horizon and $\mathbb{E}$ the expectation with respect to $\mathbb{P}_{\circ}$. As is well known, if no boundedness or integrability conditions were placed on the investment strategies, then any distribution at terminal time could be attained.

Our agent wants to choose a strategy $u$ in order to maximize expected return over a fixed time interval $[0, T]$, while trying at the same time to minimize financial risk. Interpreting risk as the variance of the underlying process and switching from gains to be maximized to costs to be minimized, the optimization problem is therefore to

$$
\begin{aligned}
& \text { minimize } J(u) \doteq \lambda \operatorname{Var}(X(T))-\mathbb{E}(X(T)) \\
& \text { subject to }\left\{\begin{array}{l}
u \in H_{T}^{2}\left(\left(\mathcal{F}_{t}^{\circ}\right)\right), \\
X \text { satisfies equation }(2.2) \text { with strategy } u \text { and } X(0)=x_{0} .
\end{array}\right.
\end{aligned}
$$

In (2.3), $\lambda>0$ is a fixed parameter, the risk aversion parameter. A strategy $\bar{u} \in H_{T}^{2}\left(\left(\mathcal{F}_{t}^{\circ}\right)\right)$ is called optimal if $J(\bar{u})=\inf _{u \in H_{T}^{2}\left(\left(\mathcal{F}_{t}^{\circ}\right)\right)} J(u)$. A function $\bar{z}:[0, T] \times \mathbb{R} \rightarrow \mathbb{R}$ is called an optimal feedback control if the equation

$$
\bar{X}(t)=x_{0}+\mu \int_{0}^{t} \bar{z}(s, \bar{X}(s)) \mathrm{d} t+\sigma \int_{0}^{t} \bar{z}(s, \bar{X}(s)) \mathrm{d} W(t), \quad t \in[0, T],
$$

possesses a unique strong solution $\bar{X}$ such that

$$
\bar{u}(t, \omega) \doteq \bar{z}(t, \bar{X}(t, \omega)), \quad t \in[0, T], \omega \in \Omega_{\circ},
$$

defines an optimal strategy, that is, $\bar{u} \in H_{T}^{2}\left(\left(\mathcal{F}_{t}^{\circ}\right)\right)$ and $J(\bar{u})=\inf _{u \in H_{T}^{2}\left(\left(\mathcal{F}_{t}^{\circ}\right)\right)} J(u)$. The strategy $\bar{u}$ will be referred to as the strategy induced by $\bar{z}$, and the process $\bar{X}$ will be referred to as the portfolio process induced by $\bar{z}$. Notice that both $\bar{u}$ and $\bar{X}$ depend on $\bar{z}$ as well as the driving Wiener process.

\section{Auxiliary prelimit optimization problems}

Let $K \in \mathbb{N} \backslash\{1\}$, and let $W_{j}, j \in\{1, \ldots, K\}$, be $K$ independent standard one-dimensional Wiener processes defined on some filtered probability space $\left(\Omega, \mathcal{F},\left(\mathcal{F}_{t}\right), \mathbb{P}\right)$. The processes $W_{j}$ can be interpreted as independent clones of the Wiener process in (2.1). For $j \in\{1, \ldots, K\}$, define $S_{j}$ according to equation (2.1) with $W$ replaced by $W_{j}$. If $u_{j}$ is a real-valued $\left(\mathcal{F}_{t}\right)$-adapted process, then the value of the agent's portfolio in market clone $j$ follows the dynamics

$$
\mathrm{d} X_{j}(t)=\mu u_{j}(t) \mathrm{d} t+\sigma u_{j}(t) \mathrm{d} W_{j}(t) .
$$

The $\mathbb{R}^{K}$-valued process $\boldsymbol{X}=\left(X_{1}, \ldots, X_{K}\right)^{\top}$ (we use boldface symbols for $K$-dimensional column vectors) thus obeys the stochastic differential equation

$$
\mathrm{d} \boldsymbol{X}(t)=\mu \boldsymbol{u}(t) \mathrm{d} t+\sigma \operatorname{diag}\left(u_{1}(t), \ldots, u_{K}(t)\right) \mathrm{d} \boldsymbol{W}(t),
$$

where $\boldsymbol{u}=\left(u_{1}, \ldots, u_{K}\right)^{\top}$ is the agent's investment strategy for the $K$ market clones and $\boldsymbol{W}=\left(W_{1}, \ldots, W_{K}\right)^{\top}$. Clearly, $\boldsymbol{W}$ is a $K$-dimensional standard Wiener process.

Given a vector $\boldsymbol{v} \in \mathbb{R}^{K}$, the empirical measure associated with $\boldsymbol{v}$ is the probability measure on the Borel sets of $\mathbb{R}$ given by

$$
\mu_{\boldsymbol{v}}^{K} \doteq \frac{1}{K} \sum_{j=1}^{K} \delta_{v_{j}},
$$

where $\delta_{x}$ denotes Dirac measure concentrated in $x \in \mathbb{R}$. The empirical mean of a $K$-dimensional vector is the mean calculated with respect to its empirical measure, which equals the arithmetic mean:

$$
\mathrm{em}(\boldsymbol{v}) \doteq \int_{\mathbb{R}} y \mu_{\boldsymbol{v}}^{K}(\mathrm{~d} y)=\frac{1}{K} \sum_{i=1}^{K} v_{j} .
$$


Similarly, the empirical variance of a $K$-dimensional vector is the variance from the empirical mean calculated with respect to the empirical measure:

$$
\begin{aligned}
\operatorname{emp} \operatorname{Var}(\boldsymbol{v}) & \doteq \int_{\mathbb{R}}(y-\operatorname{em}(\boldsymbol{v}))^{2} \mu_{\boldsymbol{v}}^{K}(\mathrm{~d} y) \\
& =\frac{1}{K} \sum_{j=1}^{K}\left(v_{j}-\mathrm{em}(\boldsymbol{v})\right)^{2} \\
& =\operatorname{em}\left(\boldsymbol{v}^{2}\right)-\operatorname{em}(\boldsymbol{v})^{2}
\end{aligned}
$$

The square in $\boldsymbol{v}^{2}$ is to be understood component-wise, that is, $\boldsymbol{v}^{2}$ is the $K$-dimensional vector $\left(v_{1}^{2}, \ldots, v_{K}^{2}\right)^{\top}$.

Recall that $T$ is the finite time horizon. In setting up the $K$-clone optimization problem, it will be convenient to allow the initial time and the initial state to vary. In addition, we adopt the weak formulation of a stochastic control problem, that is, the stochastic basis and the driving noise processes are not fixed but part of the control. To be more precise, let $\mathcal{U}_{K}$ be the set of all triples $\left(\left(\Omega, \mathcal{F},\left(\mathcal{F}_{t}\right), \mathbb{P}\right), \boldsymbol{W}, \boldsymbol{u}\right)$ such that $\left(\Omega, \mathcal{F},\left(\mathcal{F}_{t}\right), \mathbb{P}\right)$ is a filtered probability space satisfying the usual hypotheses, $\boldsymbol{W}$ is a $K$-dimensional standard $\left(\mathcal{F}_{t}\right)$-Wiener process, and $\boldsymbol{u}=\left(u_{1}, \ldots, u_{K}\right)^{\top}$ is such that $u_{j} \in H_{T}^{2}\left(\left(\mathcal{F}_{t}\right)\right)$ for every $j \in\{1, \ldots, K\}$. With a slight abuse of notation, we will occasionally write $\boldsymbol{u} \in \mathcal{U}_{K}$ instead of $\left(\left(\Omega, \mathcal{F},\left(\mathcal{F}_{t}\right), \mathbb{P}\right), \boldsymbol{W}, \boldsymbol{u}\right) \in \mathcal{U}_{K}$. For $(t, \boldsymbol{x}) \in[0, T] \times \mathbb{R}^{K}$, the $K$-clone optimization problem is to

$$
\begin{aligned}
& \operatorname{minimize} J_{K}(t, \boldsymbol{x} ; \boldsymbol{u}) \doteq \lambda \mathbb{E}\left(\mathrm{em}\left(\boldsymbol{X}(T)^{2}\right)-\mathrm{em}(\boldsymbol{X}(T))^{2}\right)-\mathbb{E}(\mathrm{em}(\boldsymbol{X}(T))) \\
& \text { subject to }\left\{\begin{array}{l}
\boldsymbol{u} \in \mathcal{U}_{K}, \\
\boldsymbol{X} \text { solves equation (3.2) with strategy } \boldsymbol{u} \text { and } \boldsymbol{X}(t)=\boldsymbol{x} .
\end{array}\right.
\end{aligned}
$$

The value function associated with (3.3) is defined as

$$
V_{K}(t, \boldsymbol{x}) \doteq \inf _{\boldsymbol{u} \in \mathcal{U}_{K}} J_{K}(t, \boldsymbol{x} ; \boldsymbol{u}), \quad(t, \boldsymbol{x}) \in[0, T] \times \mathbb{R}^{K}
$$

Theorem 3.1. The value function for the optimization problem (3.3) is given by

$$
V_{K}(t, \boldsymbol{x})=\frac{1}{4 \lambda}\left(1-\mathrm{e}^{\frac{\mu^{2}(T-t)}{\sigma^{2}(1-1 / K)}}\right)-\operatorname{em}(\boldsymbol{x})+\lambda \mathrm{e}^{-\frac{\mu^{2}(T-t)}{\sigma^{2}(1-1 / K)}}\left(\mathrm{em}\left(\boldsymbol{x}^{2}\right)-\mathrm{em}(\boldsymbol{x})^{2}\right),
$$

and the function $\overline{\boldsymbol{z}}^{(K)}:[0, T] \times \mathbb{R}^{K} \rightarrow \mathbb{R}^{K}$ defined by

$$
\bar{z}_{j}^{(K)}(t, \boldsymbol{x}) \doteq \frac{\mu}{\sigma^{2}(1-1 / K)}\left(\mathrm{em}(\boldsymbol{x})-x_{j}+\frac{1}{2 \lambda} \mathrm{e}^{\frac{\mu^{2}(T-t)}{\sigma^{2}(1-1 / K)}}\right), \quad j \in\{1, \ldots, K\},
$$

yields an optimal feedback control.

Proof. The cost functional $J_{K}$ is linear-quadratic in the state vector $\boldsymbol{X}(T)$. To be more precise, define a $K$ dimensional vector $c$ and a $K \times K$ symmetric matrix $G$ by

$$
c \doteq \frac{1}{K}\left[\begin{array}{c}
1 \\
\vdots \\
1
\end{array}\right], \quad G \doteq \frac{2 \lambda}{K^{2}}\left[\begin{array}{ccccc}
K-1 & -1 & -1 & \cdots & -1 \\
-1 & K-1 & -1 & \cdots & -1 \\
-1 & -1 & K-1 & \cdots & -1 \\
\vdots & \vdots & \vdots & \ddots & -1 \\
-1 & -1 & -1 & -1 & K-1
\end{array}\right] .
$$


Then

$$
J_{K}(t, \boldsymbol{x} ; \boldsymbol{u})=\mathbb{E}\left(\frac{1}{2}\langle G \boldsymbol{X}(T), \boldsymbol{X}(T)\rangle+\langle\boldsymbol{c}, \boldsymbol{X}(T)\rangle\right) .
$$

The controlled dynamics, which are given by equation (3.2), can be rewritten as

$$
\mathrm{d} \boldsymbol{X}(t)=\boldsymbol{b}(t, \boldsymbol{X}(t), \boldsymbol{u}(t)) \mathrm{d} t+\Sigma(t, \boldsymbol{X}(t), \boldsymbol{u}(t)) \mathrm{d} \boldsymbol{W}(t)
$$

with functions $\boldsymbol{b}:[0, \infty) \times \mathbb{R}^{K} \times \mathbb{R}^{K} \rightarrow \mathbb{R}^{K}, \Sigma:[0, \infty) \times \mathbb{R}^{K} \times \mathbb{R}^{K} \rightarrow \mathbb{R}^{K \times K}$ defined as

$$
b_{j}(t, \boldsymbol{x}, \gamma) \doteq \mu \gamma_{j}, \quad \Sigma_{i j}(t, \boldsymbol{x}, \gamma) \doteq \sigma \gamma_{j} \delta_{i j}, \quad i, j \in\{1, \ldots, K\} .
$$

The control problem (3.3) is thus of the linear-quadratic type (i.e., linear-affine dynamics, linear-quadratic costs); its generalized Hamiltonian $H$ is given by

$$
H(t, \boldsymbol{x}, \boldsymbol{\gamma}, \boldsymbol{p}, P) \doteq \sum_{j=1}^{K}\left(\mu p_{j} \gamma_{j}+\frac{\sigma^{2}}{2} P_{j j} \gamma_{j}^{2}\right)
$$

By the principle of dynamic programming, the value function should solve (at least in the sense of viscosity solutions) the Hamilton-Jacobi-Bellman terminal value problem

$$
\begin{cases}-\frac{\partial}{\partial t} v-\inf _{\boldsymbol{\gamma} \in \mathbb{R}^{K}} H\left(t, \boldsymbol{x}, \boldsymbol{\gamma}, \nabla_{\boldsymbol{x}} v, D_{\boldsymbol{x} \boldsymbol{x}}^{2} v\right)=0 & \text { if }(t, \boldsymbol{x}) \in[0, T) \times \mathbb{R}^{K}, \\ v(T, \boldsymbol{x})=\frac{1}{2}\langle G \boldsymbol{x}, \boldsymbol{x}\rangle+\langle\boldsymbol{c}, \boldsymbol{x}\rangle & \text { if } t=T, \boldsymbol{x} \in \mathbb{R}^{K} .\end{cases}
$$

The static optimization problem in (3.4) can be solved explicitly whenever $P$ has non-zero diagonal entries. In fact, for every $(t, \boldsymbol{x}, \boldsymbol{p}) \in[0, T] \times \mathbb{R}^{K} \times \mathbb{R}^{K}$, every symmetric $K \times K$ matrix $P=\left(P_{i j}\right)$ with non-zero diagonal entries,

$$
\operatorname{argmin}_{\boldsymbol{\gamma} \in \mathbb{R}^{K}} H(t, \boldsymbol{x}, \boldsymbol{\gamma}, \boldsymbol{p}, P)=\left(-\frac{\mu}{\sigma^{2}} \frac{p_{1}}{P_{11}}, \ldots,-\frac{\mu}{\sigma^{2}} \frac{p_{K}}{P_{K K}}\right)^{\top} .
$$

By a standard verification theorem (for instance, Thm. III.8.1 in [9], p. 135), if $v$ is a classical solution of (3.4), that is, $v$ is in $C^{1,2}\left([0, T] \times \mathbb{R}^{K}\right)$, satisfies (3.4) in the sense of classical calculus, and $v$ as well as its first and second order partial derivatives are of at most polynomial growth, then $v$ coincides with the value function of the optimization problem (3.3). In view of the form of the controlled dynamics (linear in the control, no explicit state dependency), the linear-quadratic terminal costs, zero running costs, and the finite time horizon $T$, a good guess for $v:[0, T] \times \mathbb{R}^{K} \rightarrow \mathbb{R}^{K}$ is

$$
v(t, \boldsymbol{x})=f(t)+\mathrm{e}^{\beta(T-t)}\langle\boldsymbol{c}, \boldsymbol{x}\rangle+\frac{\mathrm{e}^{\alpha(T-t)}}{2}\langle G \boldsymbol{x}, \boldsymbol{x}\rangle
$$

for some constants $\alpha, \beta \in[0, \infty)$ and some function $f \in C^{1}([0, T])$ such that $f(T)=0$. With this ansatz, $v$ is in $C^{1,2}\left([0, T] \times \mathbb{R}^{K}\right)$ and its Hessian $D_{\boldsymbol{x} \boldsymbol{x}}^{2} v$ always has non-zero diagonal entries. Plugging $v$ into (3.4), and using (3.5), we find the unknown parameters $\alpha, \beta$, and $f(\cdot)$ according to

$$
\alpha=-\frac{\mu^{2}}{\sigma^{2}} \frac{K}{K-1}, \quad \quad \quad \quad \quad f(t)=\frac{1}{4 \lambda}\left(1-\mathrm{e}^{\frac{\mu^{2}(T-t)}{\sigma^{2}(1-1 / K)}}\right), \quad t \in[0, T] .
$$

With this choice of the parameters,

$$
v(t, \boldsymbol{x})=\frac{1}{4 \lambda}\left(1-\mathrm{e}^{\frac{\mu^{2}(T-t)}{\sigma^{2}(1-1 / K)}}\right)-\mathrm{em}(\boldsymbol{x})+\lambda \mathrm{e}^{-\frac{\mu^{2}(T-t)}{\sigma^{2}(1-1 / K)}}\left(\mathrm{em}\left(\boldsymbol{x}^{2}\right)-\mathrm{em}(\boldsymbol{x})^{2}\right),
$$


which, by the verification theorem cited above, is equal to the value function of control problem (3.3). Calculating the derivatives $\nabla_{\boldsymbol{x}} v(t, \boldsymbol{x}), D_{\boldsymbol{x} \boldsymbol{x}}^{2} v(t, \boldsymbol{x})$ with the above choice of parameters and plugging them into (3.5) yields the feedback control $\overline{\boldsymbol{z}}^{(K)}$. Notice that $\overline{\boldsymbol{z}}^{(K)}$ is Lipschitz continuous in the state vector $\boldsymbol{x}$ so that the equation

$$
\mathrm{d} \boldsymbol{X}(t)=\mu \overline{\boldsymbol{z}}^{(K)}(t, \boldsymbol{X}(t)) \mathrm{d} t+\sigma \operatorname{diag}\left(\bar{z}_{1}^{(K)}(t, \boldsymbol{X}(t)), \ldots, \bar{z}_{K}^{(K)}(t, \boldsymbol{X}(t))\right) \mathrm{d} \boldsymbol{W}(t)
$$

possesses a unique (Markovian) solution given any deterministic initial condition. It follows (cf. [9], p. 136) that $\overline{\boldsymbol{z}}^{(K)}$ is an optimal feedback control. Since the argmin in (3.5) is unique, the optimal feedback control $\overline{\boldsymbol{z}}^{(K)}$ is unique. The corresponding optimal strategy for initial condition $\boldsymbol{X}\left(t_{0}\right)=\boldsymbol{x}$ is determined by $\boldsymbol{u}(t, \omega) \doteq$ $\overline{\boldsymbol{z}}^{(K)}(t, \boldsymbol{X}(t, \omega)), t \geq t_{0}, \omega \in \Omega$; notice that $\boldsymbol{u} \in \mathcal{U}_{K}$.

Remark 3.2. In the proof of Theorem 3.1, we made a linear-quadratic ansatz for the function $v$. The nonstandard part of our guess is the choice of the matrix $G$ for the quadratic form and the vector $c$ for the linear part. The choice of $c$ comes from the symmetry of dynamics and costs with respect to the components of the state vector. Notice that there is no direct interaction between components in the dynamics of the controlled process $\boldsymbol{X}$. The choice of $G$ is also suggested by the terminal condition of the HJB equation (3.4) or, equivalently, the terminal costs in the definition of the cost functional $J_{K}$. Alternatively, though less convincingly, one can obtain a good guess for $v$ by looking at the discrete time situation studied in [2].

\section{Passage to the Limit}

We next show that, by letting the number of clones $K$ tend to infinity, we obtain a feedback control that is optimal for the original optimization problem (2.3). By Theorem 3.1, the optimal feedback control for the $K$-clone optimization problem is given by $\overline{\boldsymbol{z}}^{(K)}=\left(\bar{z}_{1}^{(K)}, \ldots, \bar{z}_{K}^{(K)}\right)^{\top}$ with

$$
\bar{z}_{j}^{(K)}(t, \boldsymbol{x})=\frac{\mu}{\sigma^{2}(1-1 / K)}\left(\mathrm{em}(\boldsymbol{x})-x_{j}+\frac{1}{2 \lambda} \mathrm{e}^{\frac{\mu^{2}(T-t)}{\sigma^{2}(1-1 / K)}}\right) .
$$

In passing to the limit, it will be convenient to work with a fixed stochastic basis carrying an infinite family of independent one-dimensional standard Wiener processes. Thus, let $\left(\Omega, \mathcal{F},\left(\mathcal{F}_{t}\right), \mathbb{P}\right)$ be a filtered probability space satisfying the usual hypotheses and carrying a sequence $\left(W_{j}\right)_{j \in \mathbb{N}}$ of independent one-dimensional $\left(\mathcal{F}_{t}\right)$-Wiener processes. Recall that $x_{0}$ is the initial state at time zero for the portfolio process of the original problem (2.3). For $K \in \mathbb{N} \backslash\{1\}$, set $\boldsymbol{x}_{0}^{(K)} \doteq\left(x_{0}, \ldots, x_{0}\right)^{\top} \in \mathbb{R}^{K}$, and let $\overline{\boldsymbol{X}}^{(K)}=\left(\bar{X}_{1}^{(K)}, \ldots, \bar{X}_{K}^{(K)}\right)^{\top}$ denote the unique strong solution of the system of stochastic differential equations

$$
\mathrm{d} \bar{X}_{j}^{(K)}(t)=\mu \bar{z}_{j}^{(K)}\left(t, \overline{\boldsymbol{X}}^{(K)}(t)\right) \mathrm{d} t+\sigma \bar{z}_{j}^{(K)}\left(t, \overline{\boldsymbol{X}}^{(K)}(t)\right) \mathrm{d} W_{j}(t),
$$

$j \in\{1, \ldots, K\}$, with initial condition $\overline{\boldsymbol{X}}^{(K)}(0)=\boldsymbol{x}_{0}^{(K)}$. The process $\overline{\boldsymbol{X}}^{(K)}$ has the same distribution as the unique solution to equation (3.2) (or the system of equations determined by (3.1)) when feedback control $\overline{\boldsymbol{z}}^{(K)}$ is applied and the initial condition is $\boldsymbol{x}_{0}^{(K)}$ at time zero; for the corresponding costs we have

$$
\lambda \mathbb{E}\left(\operatorname{em}\left(\overline{\boldsymbol{X}}^{(K)}(T)^{2}\right)-\mathrm{em}\left(\overline{\boldsymbol{X}}^{(K)}(T)\right)^{2}\right)-\mathbb{E}\left(\mathrm{em}\left(\overline{\boldsymbol{X}}^{(K)}(T)\right)\right)=V_{K}\left(0, \boldsymbol{x}_{0}^{(K)}\right) .
$$

Define the function $\bar{z}:[0, T] \times \mathbb{R} \rightarrow \mathbb{R}$ by

$$
\bar{z}(t, x) \doteq \frac{\mu}{\sigma^{2}}\left(x_{0}+\frac{1}{2 \lambda} \mathrm{e}^{\frac{\mu^{2}}{\sigma^{2}} T}-x\right) .
$$


The function $\bar{z}$ will turn out to be an optimal feedback control for the optimization problem of Section 2. Notice that $\bar{z}$ is Lipschitz continuous in the space variable. For $j \in \mathbb{N}$, let $\bar{X}_{j}$ be the unique strong solution of

$$
\bar{X}_{j}(t)=x_{0}+\mu \int_{0}^{t} \bar{z}\left(s, \bar{X}_{j}(s)\right) \mathrm{d} s+\sigma \int_{0}^{t} \bar{z}\left(s, \bar{X}_{j}(s)\right) \mathrm{d} W_{j}(s) .
$$

Lemma 4.1. Define functions $m, n:[0, T] \rightarrow \mathbb{R}$ by

$$
\begin{aligned}
& m(t) \doteq x_{0}+\frac{1}{2 \lambda}\left(\mathrm{e}^{\frac{\mu^{2}}{\sigma^{2}} T}-\mathrm{e}^{\frac{\mu^{2}}{\sigma^{2}}(T-t)}\right) \\
& n(t) \doteq x_{0}^{2}+\frac{x_{0}}{\lambda}\left(\mathrm{e}^{\frac{\mu^{2}}{\sigma^{2}} T}-\mathrm{e}^{\frac{\mu^{2}}{\sigma^{2}}(T-t)}\right)+\frac{1}{4 \lambda^{2}}\left(\mathrm{e}^{2 \frac{\mu^{2}}{\sigma^{2}} T}-\mathrm{e}^{\frac{\mu^{2}}{\sigma^{2}}(2 T-t)}\right) .
\end{aligned}
$$

Then the following convergences hold uniformly in $t \in[0, T]$ :

(a) $\lim _{K \rightarrow \infty} \operatorname{em}\left(\overline{\boldsymbol{X}}^{(K)}(t)\right)=m(t)$ in $L^{2}(\mathbb{P})$;

(b) $\lim _{K \rightarrow \infty} \mathbb{E}\left(\operatorname{em}\left(\left(\overline{\boldsymbol{X}}^{(K)}(t)\right)^{2}\right)\right)=n(t)$;

(c) for every $j \in \mathbb{N}$,

$$
\lim _{K \rightarrow \infty} \bar{z}_{j}^{(K)}\left(t, \overline{\boldsymbol{X}}^{(K)}(t)\right)=\bar{z}\left(t, \bar{X}_{j}(t)\right), \quad \quad \lim _{K \rightarrow \infty} \bar{X}_{j}^{(K)}(t)=\bar{X}_{j}(t) \text { in } L^{2}(\mathbb{P}) .
$$

Moreover, for every $j \in \mathbb{N}$, every $t \in[0, T]$,

$$
\mathbb{E}\left(\bar{X}_{j}(t)\right)=m(t), \quad \mathbb{E}\left(\left(\bar{X}_{j}(t)\right)^{2}\right)=n(t) .
$$

Proof. For $K \in \mathbb{N} \backslash\{1\}, t \in[0, T]$, set

$$
\begin{array}{ll}
Y^{(K)}(t) \doteq \mathrm{em}\left(\overline{\boldsymbol{X}}^{(K)}(t)\right), & L^{(K)}(t) \doteq \mathrm{em}\left(\overline{\boldsymbol{X}}^{(K)}(t)^{2}\right), \\
g_{K}(t) \doteq \frac{1}{2 \lambda} \mathrm{e}^{\frac{\mu^{2}}{\sigma^{2}(1-1 / K)} t}, & g(t) \doteq \frac{1}{2 \lambda} \mathrm{e}^{\frac{\mu^{2}}{\sigma^{2}} t} .
\end{array}
$$

Clearly, $C_{K} \rightarrow \mu / \sigma^{2}, g_{K}(t) \rightarrow g(t)$ uniformly in $t \in[0, T]$ as $K \rightarrow \infty$. By definition, $Y^{(K)}(t)=\frac{1}{K} \sum_{j=1}^{K} \bar{X}_{j}^{(K)}(t)$, $L^{(K)}(t)=\frac{1}{K} \sum_{j=1}^{K} \bar{X}_{j}^{(K)}(t)^{2}$. Notice that

$$
\begin{aligned}
m(t) & =x_{0}+g(T)-g(T-t) . \\
n(t) & =m(t)^{2}-g(T-t / 2)^{2}-g(T-t)^{2}+2 g(T) g(T-t) .
\end{aligned}
$$

In view of (4.1) and (4.2), $Y^{(K)}$ solves the stochastic differential equation

$$
\mathrm{d} Y^{(K)}(t)=\mu C_{K} g_{K}(T-t) \mathrm{d} t+\frac{1}{K} \sum_{j=1}^{K} \sigma C_{K}\left(Y^{(K)}(t)-\bar{X}_{j}^{(K)}(t)+g_{K}(T-t)\right) \mathrm{d} W_{j}(t)
$$

with initial condition $Y^{(K)}(0)=x_{0}$, while $L^{(K)}$ solves

$$
\begin{aligned}
\mathrm{d} L^{(K)}(t)= & 2 \mu C_{K}\left(Y^{(K)}(t)^{2}-L^{(K)}(t)+Y^{(K)}(t) g_{K}(T-t)\right) \mathrm{d} t \\
& +\sigma^{2} C_{K}^{2}\left(L^{(K)}(t)-Y^{(K)}(t)^{2}+g_{K}(T-t)^{2}\right) \mathrm{d} t \\
& +\frac{1}{K} \sum_{j=1}^{K} 2 \sigma C_{K} \bar{X}_{j}^{(K)}(t)\left(Y^{(K)}(t)-\bar{X}_{j}^{(K)}(t)+g_{K}(T-t)\right) \mathrm{d} W_{j}(t)
\end{aligned}
$$


with initial condition $L^{(K)}(0)=x_{0}^{2}$. By the independence of the Wiener processes $W_{1}, \ldots, W_{K}$, the BurkholderDavis-Gundy inequality, and Gronwall's lemma, $\bar{X}_{1}^{(K)}, \ldots, \bar{X}_{K}^{(K)}, Y^{(K)}, L^{(K)}$ possess moments of any polynomial order; moreover,

$$
\sup _{K \in \mathbb{N}} \sup _{s \in[0, T]} \mathbb{E}\left(L^{(K)}(s)\right)=\sup _{K \in \mathbb{N}} \sup _{s \in[0, T]} \mathbb{E}\left(\frac{1}{K} \sum_{j=1}^{K}\left|\bar{X}_{j}^{(K)}(s)\right|^{2}\right)<\infty .
$$

In order to prove the limit in (a), it is enough to show that

$$
\limsup _{K \rightarrow \infty} \sup _{t \in[0, T]} \mathbb{E}\left(\left|Y^{(K)}(t)-m(t)\right|^{2}\right) \leq 0 .
$$

Integration of equation (4.5) yields, for every $t \in[0, T]$,

$$
Y^{(K)}(t)=x_{0}+g_{K}(T)-g_{K}(T-t)+\frac{1}{K} \sum_{j=1}^{K} \int_{0}^{t} \sigma C_{K}\left(Y^{(K)}(s)-\bar{X}_{j}^{(K)}(s)+g_{K}(T-s)\right) \mathrm{d} W_{j}(s) .
$$

Using Itô's isometry and the independence of the Wiener processes, one finds that

$$
\begin{aligned}
\mathbb{E}\left(\left|Y^{(K)}(t)-m(t)\right|^{2}\right) \leq & 2\left(g_{K}(T)-g(T)+g(T-t)-g_{K}(T-t)\right)^{2} \\
& +\frac{2 \mu^{2}}{\sigma^{2}(K-1)^{2}} \mathbb{E}\left(\left(\sum_{j=1}^{K} \int_{0}^{t}\left(Y^{(K)}(s)-\bar{X}_{j}^{(K)}(s)+g_{K}(T-s)\right) \mathrm{d} W_{j}(s)\right)^{2}\right) \\
= & 2\left(g_{K}(T)-g(T)+g(T-t)-g_{K}(T-t)\right)^{2} \\
& +\frac{2 \mu^{2}}{\sigma^{2}(K-1)^{2}} \sum_{j=1}^{K} \int_{0}^{t} \mathbb{E}\left(\left(Y^{(K)}(s)-\bar{X}_{j}^{(K)}(s)+g_{K}(T-s)\right)^{2}\right) \mathrm{d} s \\
\leq & 2\left(g_{K}(T)-g(T)+g(T-t)-g_{K}(T-t)\right)^{2} \\
& +\frac{16 T \mu^{2}}{\sigma^{2}(K-1)^{2}}\left(K g_{K}(T)^{2}+\sup _{s \in[0, T]} \mathbb{E}\left(\sum_{j=1}^{K}\left(\bar{X}_{j}^{(K)}(s)\right)^{2}\right)\right) .
\end{aligned}
$$

Since $g_{K}(t) \rightarrow g(t)$ uniformly and thanks to (4.7),

$$
\begin{aligned}
\limsup _{K \rightarrow \infty} \sup _{t \in[0, T]} \mathbb{E}\left(\left|Y^{(K)}(t)-m(t)\right|^{2}\right) & \\
& \leq \limsup _{K \rightarrow \infty} \frac{16 T \mu^{2} K}{\sigma^{2}(K-1)^{2}}\left(g_{K}(T)^{2}+\sup _{s \in[0, T]} \mathbb{E}\left(\frac{1}{K} \sum_{j=1}^{K}\left(\bar{X}_{j}^{(K)}(s)\right)^{2}\right)\right)=0 .
\end{aligned}
$$

In order to prove (b), set

$$
n_{K}(t) \doteq \mathbb{E}\left(L^{(K)}(t)\right), \quad t \in[0, T]
$$


We have to show that $\lim _{K \rightarrow \infty} n_{K}(t)=n(t)$ for every $t \in[0, T]$. Integration of equation (4.6) yields, for $t \in[0, T]$,

$$
\begin{aligned}
L^{(K)}(t)= & x_{0}^{2}+\left(2 \mu C_{K}-\sigma^{2} C_{K}^{2}\right) \int_{0}^{t}\left(Y^{(K)}(s)^{2}-L^{(K)}(s)\right) \mathrm{d} s \\
& +2 \mu C_{K} \int_{0}^{t} Y^{(K)}(s) g_{K}(T-s) \mathrm{d} s+\sigma^{2} C_{K}^{2} \int_{0}^{t} g_{K}(T-s)^{2} \mathrm{~d} s \\
& +\frac{1}{K} \sum_{j=1}^{K} \int_{0}^{t} 2 \sigma C_{K} \bar{X}_{j}^{(K)}(s)\left(Y^{(K)}(s)-\bar{X}_{j}^{(K)}(s)+g_{K}(T-s)\right) \mathrm{d} W_{j}(s) .
\end{aligned}
$$

The stochastic integral in the above display is a true martingale thanks to the $L^{p}$-integrability of the components of $\overline{\boldsymbol{X}}^{(K)}$. Using the Fubini-Tonelli theorem, it follows that $n_{K}(\cdot)$ satisfies the integral equation

$$
\begin{aligned}
n_{K}(t)= & x_{0}^{2}+\left(2 \mu C_{K}-\sigma^{2} C_{K}^{2}\right) \int_{0}^{t}\left(\mathbb{E}\left(Y^{(K)}(s)^{2}\right)-n_{K}(s)\right) \mathrm{d} s \\
& +2 \mu C_{K} \int_{0}^{t} \mathbb{E}\left(Y^{(K)}(s)\right) g_{K}(T-s) \mathrm{d} s+\sigma^{2} C_{K}^{2} \int_{0}^{t} g_{K}(T-s)^{2} \mathrm{~d} s .
\end{aligned}
$$

Notice that $\lim _{K \rightarrow \infty} \sigma^{2} C_{K}^{2}=\frac{\mu^{2}}{\sigma^{2}}=\lim _{K \rightarrow \infty} \mu C_{K}$. Let $\bar{n}(\cdot)$ denote the unique solution over $[0, T]$ of the integral equation

$$
\bar{n}(t)=x_{0}^{2}+\frac{\mu^{2}}{\sigma^{2}} \int_{0}^{t}(m(s)+g(T-s))^{2} \mathrm{~d} s-\frac{\mu^{2}}{\sigma^{2}} \int_{0}^{t} \bar{n}(s) \mathrm{d} s .
$$

By part (a), $\mathbb{E}\left(Y^{(K)}(s)\right) \rightarrow m(s)$ and $\mathbb{E}\left(Y^{(K)}(s)^{2}\right) \rightarrow m(s)^{2}$ as $K \rightarrow \infty$, uniformly in $s \in[0, T]$. It follows that $\lim _{K \rightarrow \infty} n_{K}(t)=\bar{n}(t)$, uniformly in $t \in[0, T]$. Since $m(s)+g(T-s)=x_{0}+\frac{1}{2 \lambda} \mathrm{e}^{\frac{\mu^{2}}{\sigma^{2}} T}, \bar{n}(\cdot)$ is the unique solution over $[0, T]$ of the integral equation

$$
\bar{n}(t)=x_{0}^{2}+\frac{\mu^{2}}{\sigma^{2}} t\left(x_{0}+\frac{1}{2 \lambda} \mathrm{e}^{\frac{\mu^{2}}{\sigma^{2}} T}\right)^{2}-\frac{\mu^{2}}{\sigma^{2}} \int_{0}^{t} \bar{n}(s) \mathrm{d} s
$$

or, equivalently, the unique solution over $[0, T]$ of the differential equation

$$
\frac{\mathrm{d}}{\mathrm{d} t} \bar{n}(t)=\frac{\mu^{2}}{\sigma^{2}}\left(x_{0}+\frac{1}{2 \lambda} \mathrm{e}^{\frac{\mu^{2}}{\sigma^{2}} T}\right)^{2}-\frac{\mu^{2}}{\sigma^{2}} \bar{n}(t)
$$

with initial condition $\bar{n}(0)=x_{0}^{2}$. That solution is given by

$$
\begin{aligned}
\bar{n}(t) & =\mathrm{e}^{-\frac{\mu^{2}}{\sigma^{2}} t}\left(x_{0}^{2}+\frac{\mu^{2}}{\sigma^{2}}\left(x_{0}+\frac{1}{2 \lambda} \mathrm{e}^{\frac{\mu^{2}}{\sigma^{2}} T}\right)^{2} \int_{0}^{t} \mathrm{e}^{\frac{\mu^{2}}{\sigma^{2}} s} \mathrm{~d} s\right) \\
& =x_{0}^{2}+\frac{x_{0}}{\lambda}\left(\mathrm{e}^{\frac{\mu^{2}}{\sigma^{2}} T}-\mathrm{e}^{\frac{\mu^{2}}{\sigma^{2}}(T-t)}\right)+\frac{1}{4 \lambda^{2}}\left(\mathrm{e}^{2 \frac{\mu^{2}}{\sigma^{2}} T}-\mathrm{e}^{\frac{\mu^{2}}{\sigma^{2}}(2 T-t)}\right) .
\end{aligned}
$$

Therefore, $\lim _{K \rightarrow \infty} n_{K}(t)=\bar{n}(t)=n(t)$, uniformly in $t \in[0, T]$.

For part (c), fix $j \in \mathbb{N}$, and let $\bar{X}_{j}$ be the unique solution of equation (4.4) with $\bar{X}_{j}(0)=x_{0}$ and driving Wiener process $W_{j}$. In order to prove (c), it is enough to show that

$$
\limsup _{K \rightarrow \infty} \sup _{t \in[0, T]} \mathbb{E}\left(\left|\bar{z}_{j}^{(K)}\left(t, \overline{\boldsymbol{X}}^{(K)}(t)\right)-\bar{z}\left(t, \bar{X}_{j}(t)\right)\right|^{2}\right) \leq 0,
$$


and analogously for $\bar{X}_{j}^{(K)}(t)$ and $\bar{X}_{j}(t)$. For $t \in[0, T], K \in \mathbb{N}$, set

$$
R_{K}(t) \doteq \frac{\mu}{\sigma^{2}(1-1 / K)}\left(\mathrm{em}\left(\overline{\boldsymbol{X}}_{j}^{K}(t)\right)+g_{K}(T-t)\right)-\frac{\mu}{\sigma^{2}}\left(x_{0}+g(T)\right)+\frac{\mu}{\sigma^{2}(K-1)} \bar{X}_{j}^{(K)}(t) .
$$

Clearly, $\lim _{K \rightarrow \infty} \frac{\mu}{\sigma^{2}(1-1 / K)}=\frac{\mu}{\sigma^{2}}$. Thanks to part (a),

$$
\mathrm{em}\left(\overline{\boldsymbol{X}}^{(K)}(t)\right)+g_{K}(T-t) \stackrel{K \rightarrow \infty}{\longrightarrow} m(t)+g(T-t)=x_{0}+g(T)
$$

in $L^{2}(\mathbb{P})$, uniformly in $t \in[0, T]$. By the symmetry of equation (4.2) and the initial condition, $\bar{X}_{1}^{(K)}(t), \ldots, \bar{X}_{K}^{(K)}(t)$ have the same distribution for every $t \in[0, T]$. Estimate (4.7) therefore implies that

$$
\sup _{K \in \mathbb{N}} \sup _{t \in[0, T]} \mathbb{E}\left(\left|\bar{X}_{j}^{(K)}(t)\right|^{2}\right)<\infty
$$

It follows that

$$
\sup _{t \in[0, T]} \mathbb{E}\left(\left|R_{K}(t)\right|^{2}\right) \stackrel{K \rightarrow \infty}{\longrightarrow} 0
$$

Now, for every $t \in[0, T]$,

$$
\begin{aligned}
\left|\bar{z}_{j}^{(K)}\left(t, \overline{\boldsymbol{X}}^{(K)}(t)\right)-\bar{z}\left(t, \bar{X}_{j}(t)\right)\right|^{2} & =\left|\frac{\mu}{\sigma^{2}}\left(\bar{X}_{j}^{(K)}(t)-\bar{X}_{j}(t)\right)+R_{K}(t)\right|^{2} \\
& \leq 2 \frac{\mu^{2}}{\sigma^{4}}\left|\bar{X}_{j}^{(K)}(t)-\bar{X}_{j}(t)\right|^{2}+2\left|R_{K}(t)\right|^{2}
\end{aligned}
$$

In view of equations (4.2) and (4.4), respectively, using Hölder's inequality, Itô's isometry, and the Fubini-Tonelli theorem, we have

$$
\begin{aligned}
\mathbb{E}\left(\left|\bar{X}_{j}^{(K)}(t)-\bar{X}_{j}(t)\right|^{2}\right) \leq & 2 \mu^{2} \mathbb{E}\left(\left(\int_{0}^{t}\left(\bar{z}_{j}^{(K)}\left(s, \overline{\boldsymbol{X}}^{(K)}(s)\right)-\bar{z}\left(s, \bar{X}_{j}(s)\right)\right) \mathrm{d} s\right)^{2}\right) \\
& +2 \sigma^{2} \mathbb{E}\left(\left(\int_{0}^{t}\left(\bar{z}_{j}^{(K)}\left(s, \overline{\boldsymbol{X}}^{(K)}(s)\right)-\bar{z}\left(s, \bar{X}_{j}(s)\right)\right) \mathrm{d} W_{j}(s)\right)^{2}\right) \\
\leq & 2\left(\mu^{2} T+\sigma^{2}\right) \int_{0}^{t} \mathbb{E}\left(\left|\bar{z}_{j}^{(K)}\left(s, \overline{\boldsymbol{X}}^{(K)}(s)\right)-\bar{z}\left(s, \bar{X}_{j}(s)\right)\right|^{2}\right) \mathrm{d} s .
\end{aligned}
$$

It follows that, for every $t \in[0, T]$,

$$
\begin{aligned}
& \mathbb{E}\left(\left|\bar{z}_{j}^{(K)}\left(t, \overline{\boldsymbol{X}}^{(K)}(t)\right)-\bar{z}\left(t, \bar{X}_{j}(t)\right)\right|^{2}\right) \\
& \quad \leq 4\left(\frac{\mu^{4}}{\sigma^{4}} T+\frac{\mu^{2}}{\sigma^{2}}\right) \int_{0}^{t} \mathbb{E}\left(\left|\bar{z}_{j}^{(K)}\left(s, \overline{\boldsymbol{X}}^{(K)}(s)\right)-\bar{z}\left(s, \bar{X}_{j}(s)\right)\right|^{2}\right) \mathrm{d} s+2 \sup _{s \in[0, T]} \mathbb{E}\left(\left|R_{K}(s)\right|^{2}\right) .
\end{aligned}
$$

Therefore, by Gronwall's lemma,

$$
\sup _{t \in[0, T]} \mathbb{E}\left(\left|\bar{z}_{j}^{(K)}\left(t, \overline{\boldsymbol{X}}^{(K)}(t)\right)-\bar{z}\left(t, \bar{X}_{j}(t)\right)\right|^{2}\right) \leq 2 \sup _{t \in[0, T]} \mathbb{E}\left(\left|R_{K}(t)\right|^{2}\right) \mathrm{e}^{4\left(\frac{\mu^{4}}{\sigma^{4}} T+\frac{\mu^{2}}{\sigma^{2}}\right) T} .
$$

Since $\sup _{t \in[0, T]} \mathbb{E}\left(\left|R_{K}(t)\right|^{2}\right) \rightarrow 0$ as $K \rightarrow \infty$, we have

$$
\limsup _{K \rightarrow \infty} \sup _{t \in[0, T]} \mathbb{E}\left(\left|\bar{z}_{j}^{(K)}\left(t, \overline{\boldsymbol{X}}^{(K)}(t)\right)-\bar{z}\left(t, \bar{X}_{j}(t)\right)\right|^{2}\right) \leq 0 .
$$


Similarly, for every $t \in[0, T]$,

$$
\begin{aligned}
& \mathbb{E}\left(\left|\bar{X}_{j}^{(K)}(t)-\bar{X}_{j}(t)\right|^{2}\right) \\
& \quad \leq 4\left(\frac{\mu^{4}}{\sigma^{4}} T+\frac{\mu^{2}}{\sigma^{2}}\right) \int_{0}^{t} \mathbb{E}\left(\left|\bar{X}^{(K)}(s)-\bar{X}_{j}(s)\right|^{2}\right) \mathrm{d} s+4 T\left(\mu^{2} T+\sigma^{2}\right) \sup _{s \in[0, T]} \mathbb{E}\left(\left|R_{K}(s)\right|^{2}\right) .
\end{aligned}
$$

Thus, again by Gronwall's lemma and since $\sup _{t \in[0, T]} \mathbb{E}\left(\left|R_{K}(t)\right|^{2}\right) \rightarrow 0$,

$$
\limsup _{K \rightarrow \infty} \sup _{t \in[0, T]} \mathbb{E}\left(\left|\bar{X}_{j}^{(K)}(t)-\bar{X}_{j}(t)\right|^{2}\right) \leq 0
$$

The last part of the assertion is now a consequence of parts (a)-(c), and the fact that $\overline{\boldsymbol{X}}^{(K)}$ has identically distributed components for every $K \in \mathbb{N}$. Alternatively, the first and second moments of $\bar{X}_{j}(t)$ can be calculated directly from equations (4.4) and (4.3), the definition of $\bar{z}$.

Theorem 4.2. The function

$$
\bar{z}(t, x) \doteq \frac{\mu}{\sigma^{2}}\left(x_{0}+\frac{1}{2 \lambda} \mathrm{e}^{\frac{\mu^{2}}{\sigma^{2}} T}-x\right), \quad(t, x) \in[0, T] \times \mathbb{R},
$$

yields an optimal feedback control for the original optimization problem (2.3), and the minimal costs are given by

$$
\lambda \operatorname{Var}(\bar{X}(T))-\mathbb{E}(\bar{X}(T))=\frac{1}{4 \lambda}\left(1-\mathrm{e}^{\frac{\mu^{2}}{\sigma^{2}} T}\right)-x_{0},
$$

where $\bar{X}$ is the portfolio process induced by $\bar{z}$.

Before giving the proof of Theorem 4.2, we make a few remarks regarding the optimal feedback control $\bar{z}$.

Remark 4.3. The optimal feedback control $\bar{z}$ is affine-linear in the current state $x$, does not depend on the current time $t$, while it depends on the time horizon $T$ as well as the initial state $x_{0}$. This last dependence is due to the non-linear nature of the cost functional, which makes the optimization problem inconsistent in time. The fact that $\bar{z}$ does not depend on the current time $t$ is something of a coincidence, due to our choice of interest rates equal to zero. Recall that, by Lemma 4.1,

$$
m(t) \doteq x_{0}+\frac{1}{2 \lambda}\left(\mathrm{e}^{\frac{\mu^{2}}{\sigma^{2}} T}-\mathrm{e}^{\frac{\mu^{2}}{\sigma^{2}}(T-t)}\right)
$$

is the $L^{2}$-limit of the empirical means of the $K$-clone optimization problem. For the optimal strategy $\bar{z}$, we can therefore write

$$
\bar{z}(t, x)=\frac{\mu}{\sigma^{2}}\left(m(t)+\frac{1}{2 \lambda} \mathrm{e}^{\frac{\mu^{2}}{\sigma^{2}}(T-t)}-x\right) .
$$

This expression coincides, in the limit as $K \rightarrow \infty$, with the expression for the optimal feedback strategy $\bar{z}_{j}^{(K)}$ established in Theorem 3.1 for the $j$ th component of the $K$-clone optimization problem. In (4.8), $\bar{z}$ apparently depends on current time, but this dependence cancels out when we plug in the expression for $m(t)$. On the other hand, $\bar{z}$ truly depends - through $m(\cdot)$ - on the initial state $x_{0}$, since $m(0)=x_{0}$. Also notice that $m(t)=\mathbb{E}(\bar{X}(t))$, where $\bar{X}$ is the optimal portfolio process, the one induced by $\bar{z}$. 
Remark 4.4. Expression (4.8) coincides with the optimal feedback strategy found at the end of Section 5 of [1] in the special case of zero interest rate $\left(\rho_{t} \equiv 0\right)$, constant volatility $\left(\sigma_{t} \equiv \sigma\right)$, and constant average rate of return $\left(\alpha_{t} \equiv \mu\right)$; our risk aversion parameter $\lambda$ corresponds to $\gamma / 2$ there. In Section 5 of [1], the price of the risky asset follows a geometric Brownian motion, not an arithmetic as here, but the resulting mean-variance optimization problem, including the dynamics for the portfolio process, is the same as in our case. The difference lies entirely in the different interpretation of the investment strategies, namely as control expressed in terms of (real-valued) number of assets versus market value of assets.

Remark 4.5. The solution to the mean-variance optimization problem seen above is known as precommitment solution. This refers to the fact that we solve the optimization problem exclusively from the point of view of the agent's state at time zero, ignoring intermediate times. The game-theoretic approach leads to a different interpretation of the optimization problem and to a different solution. The optimal feedback strategy found in $[4,5]$ for the special case of constant interest rate $r$, constant volatility $\sigma$, constant average rate of return $(\alpha=\mu)$, and constant risk aversion parameter $(\gamma / 2=\lambda)$ is given by $\hat{z}(t, x)=\frac{\mu-r}{\sigma^{2}} \frac{1}{2 \lambda} \mathrm{e}^{r(T-t)}$. In particular, if $r=0$, then

$$
\hat{z}(t, x) \equiv \frac{\mu}{\sigma^{2}} \frac{1}{2 \lambda} .
$$

It would therefore be optimal to hold a constant number of assets (Bachelier dynamics, arithmetic Brownian motion) or a constant market value of assets (Merton-Black-Scholes dynamics, geometric Brownian motion), independently of current or initial wealth. This is in stark contrast with (4.3) and (4.8), respectively.

Proof of Theorem 4.2. The strategies and portfolio processes of the original optimization problem (2.3) are all defined on the filtered probability space $\left(\Omega_{\circ}, \mathcal{F}^{\circ},\left(\mathcal{F}_{t}^{\circ}\right), \mathbb{P}_{\circ}\right)$ with $W$ an $\left(\mathcal{F}_{t}^{\circ}\right)$-Wiener process. Let $\bar{X}$ be the portfolio process induced by $\bar{z}$, that is, $\bar{X}$ is the unique strong solution of equation (2.4):

$$
\bar{X}(t)=x_{0}+\mu \int_{0}^{t} \bar{z}(s, \bar{X}(s)) \mathrm{d} s+\sigma \int_{0}^{t} \bar{z}(s, \bar{X}(s)) \mathrm{d} W(s), \quad t \in[0, T] .
$$

Let $\bar{u}$ be the strategy induced by $\bar{z}$ :

$$
\bar{u}(t, \omega) \doteq \bar{z}(t, \bar{X}(t, \omega)), \quad t \in[0, T], \omega \in \Omega .
$$

Then $\bar{u}$ is a real-valued square-integrable process with continuous trajectories, and $\bar{u}$ is adapted to the filtration generated by the Wiener process $W$. It follows that $\bar{u} \in H_{T}^{2}\left(\left(\mathcal{F}_{t}^{\circ}\right)\right)$. Moreover, $\bar{X}$ coincides with the unique solution of equation (2.2) with strategy $u=\bar{u}$ and initial condition $\bar{X}(0)=x_{0}$. By definition,

$$
J(\bar{u})=\lambda\left(\mathbb{E}\left(\bar{X}(T)^{2}\right)-\mathbb{E}(\bar{X}(T))^{2}\right)-\mathbb{E}(\bar{X}(T)) .
$$

Let $\tilde{u} \in H_{T}^{2}\left(\left(\mathcal{F}_{t}^{\circ}\right)\right)$ be any admissible strategy, and let $\tilde{X}$ be the unique solution of equation (2.2) with strategy $u=\tilde{u}$ and initial condition $\tilde{X}(0)=x_{0}$. To prove the statement, we have to show that $J(\bar{u})=\frac{1}{4 \lambda}\left(\mathrm{e}^{\frac{\mu^{2}}{\sigma^{2}} T}-1\right)-x_{0}$ and that $J(\bar{u}) \leq J(\tilde{u})$. Set

$$
\Omega \doteq \times_{i=1}^{\infty} \Omega_{\circ}, \quad \mathcal{F} \doteq \otimes_{i=1}^{\infty} \mathcal{F}^{\circ}, \quad \mathcal{F}_{t} \doteq \otimes_{i=1}^{\infty} \mathcal{F}_{t}^{\circ}, t \geq 0, \quad \mathbb{P}=\otimes_{i=1}^{\infty} \mathbb{P}_{\circ}
$$

Then $\left(\Omega, \mathcal{F},\left(\mathcal{F}_{t}\right), \mathbb{P}\right)$ is a filtered probability space satisfying the usual hypotheses. For every $j \in \mathbb{N}$, define processes $W_{j}, \bar{u}_{j}, \bar{X}_{j}, \tilde{u}_{j}, \tilde{X}_{j}$ by setting, for $t \geq 0, \omega=\left(\omega_{i}\right)_{i \in \mathbb{N}} \in \Omega$,

$$
\begin{array}{lll}
W_{j}(t, \omega) \doteq W\left(t, \omega_{j}\right), & \bar{u}_{j}(t, \omega) \doteq \bar{u}\left(t, \omega_{j}\right), & \bar{X}_{j}(t, \omega) \doteq \bar{X}\left(t, \omega_{j}\right), \\
& \tilde{u}_{j}(t, \omega) \doteq \tilde{u}\left(t, \omega_{j}\right), & \tilde{X}_{j}(t, \omega) \doteq \tilde{X}\left(t, \omega_{j}\right) .
\end{array}
$$


All processes thus defined are $\left(\mathcal{F}_{t}\right)$-progressively measurable, and $W_{1}, W_{2}, \ldots$ are independent standard $\left(\mathcal{F}_{t}\right)$ Wiener processes. The processes $W_{j}, \bar{u}_{j}, \bar{X}_{j}, \tilde{u}_{j}, \tilde{X}_{j}, j \in \mathbb{N}$, are i.i.d. copies of the processes $W, \bar{u}, \bar{X}, \tilde{u}, \tilde{X}$. More precisely, $\left(W_{j}, \bar{u}_{j}, \bar{X}_{j}, \tilde{u}_{j}, \tilde{X}_{j}\right)_{j \in \mathbb{N}}$ is a family of independent and identically distributed $\mathbb{R}^{5}$-valued processes living on $(\Omega, \mathcal{F}, \mathbb{P})$, and for every $j \in \mathbb{N}$,

$$
\mathbb{P} \circ\left(W_{j}, \bar{u}_{j}, \bar{X}_{j}, \tilde{u}_{j}, \tilde{X}_{j}\right)^{-1}=\mathbb{P}_{\circ} \circ(W, \bar{u}, \bar{X}, \tilde{u}, \tilde{X})^{-1} .
$$

In fact, $\bar{X}_{j}, \tilde{X}_{j}$ solve equation (2.2) with Wiener process $W_{j}$ in place of $W$, initial condition $x_{0}$ at time zero, and strategy $u=\bar{u}$ and $u=\tilde{u}$, respectively. Moreover, $\bar{X}_{j}$ solves equation (4.4). By Lemma 4.1, it follows that

$$
\begin{aligned}
J(\bar{u}) & =\lambda\left(\mathbb{E}\left(\bar{X}_{1}(T)^{2}\right)-\mathbb{E}\left(\bar{X}_{1}(T)\right)^{2}\right)-\mathbb{E}\left(\bar{X}_{1}(T)\right) \\
& =\lambda\left(n(T)-m(T)^{2}\right)-m(T) \\
& =\frac{1}{4 \lambda}\left(1-\mathrm{e}^{\frac{\mu^{2}}{\sigma^{2}} T}\right)-x_{0} .
\end{aligned}
$$

It remains to show that $J(\tilde{u}) \geq J(\bar{u})$. Observe that

$$
J(\bar{u})=\lim _{K \rightarrow \infty} V_{K}\left(0, x_{0}^{(K)}\right),
$$

where $V_{K}$ is the value function of Theorem 3.1 and $\boldsymbol{x}_{0}^{(K)}=\left(x_{0}, \ldots, x_{0}\right)^{\top} \in \mathbb{R}^{K}$ as above. By construction,

$$
J(\tilde{u})=\lambda\left(\mathbb{E}\left(\tilde{X}_{1}(T)^{2}\right)-\mathbb{E}\left(\tilde{X}_{1}(T)\right)^{2}\right)-\mathbb{E}\left(\tilde{X}_{1}(T)\right) .
$$

For $K \in \mathbb{N} \backslash\{1\}$, set $\tilde{\boldsymbol{X}}^{(K)} \doteq\left(\tilde{X}_{1}, \ldots, \tilde{X}_{K}\right)^{\top}$ and $\tilde{\boldsymbol{u}}^{(K)} \doteq\left(\tilde{u}_{1}, \ldots, \tilde{u}_{K}\right)^{\top}$. Then

$$
\left(\left(\Omega, \mathcal{F},\left(\mathcal{F}_{t}\right), \mathbb{P}\right),\left(W_{1}, \ldots, W_{K}\right)^{\top}, \tilde{\boldsymbol{u}}^{(K)}\right) \in \mathcal{U}_{K}
$$

and $\tilde{\boldsymbol{X}}^{(K)}$ solves equation (3.2) with strategy $\boldsymbol{u}=\tilde{\boldsymbol{u}}^{(K)}$ and initial condition $\tilde{\boldsymbol{X}}^{(K)}(0)=\boldsymbol{x}_{0}^{(K)}$. Recalling the definition of the cost functional in (3.3), we have

$$
J_{K}\left(0, \boldsymbol{x}_{0}^{(K)} ; \tilde{\boldsymbol{u}}^{(K)}\right)=\lambda \mathbb{E}\left(\mathrm{em}\left(\tilde{\boldsymbol{X}}^{(K)}(T)^{2}\right)-\mathrm{em}\left(\tilde{\boldsymbol{X}}^{(K)}(T)\right)^{2}\right)-\mathbb{E}\left(\mathrm{em}\left(\tilde{\boldsymbol{X}}^{(K)}(T)\right)\right) .
$$

By construction, $\left(\tilde{X}_{j}(T)\right)_{j \in \mathbb{N}}$ is an i.i.d. sequence of real-valued square-integrable random variables on $(\Omega, \mathcal{F}, \mathbb{P})$. Therefore,

$$
\mathbb{E}\left(\operatorname{em}\left(\tilde{\boldsymbol{X}}^{(K)}(T)\right)\right)=\mathbb{E}\left(\tilde{X}_{1}(T)\right), \quad \mathbb{E}\left(\mathrm{em}\left(\tilde{\boldsymbol{X}}^{(K)}(T)^{2}\right)\right)=\mathbb{E}\left(\tilde{X}_{1}(T)^{2}\right),
$$

while

$$
\mathbb{E}\left(\mathrm{em}\left(\tilde{\boldsymbol{X}}^{(K)}(T)\right)^{2}\right)=\frac{K(K-1)}{K^{2}} \mathbb{E}\left(\tilde{X}_{1}(T)\right)^{2}+\frac{K}{K^{2}} \mathbb{E}\left(\tilde{X}_{1}(T)^{2}\right)
$$

It follows that

$$
\lim _{K \rightarrow \infty} J_{K}\left(0, \boldsymbol{x}_{0}^{(K)} ; \tilde{\boldsymbol{u}}^{(K)}\right)=J(\tilde{u}) .
$$

Since $J_{K}\left(0, \boldsymbol{x}_{0}^{(K)} ; \tilde{\boldsymbol{u}}^{(K)}\right) \geq V_{K}\left(0, \boldsymbol{x}_{0}^{(K)}\right)$ for every $K \in \mathbb{N} \backslash\{1\}$ by definition of the value function and $\lim _{K \rightarrow \infty} V_{K}\left(0, \boldsymbol{x}_{0}^{(K)}\right)=J(\bar{u})$, we conclude that $J(\tilde{u}) \geq J(\bar{u})$.

Acknowledgements. The authors are grateful to three anonymous referees for their critique and many helpful suggestions. 


\section{REFERENCES}

[1] D. Andersson and B. Djehiche, A maximum principle for SDEs of mean-field type. Appl. Math. Optim. 63 (2011) 341-356.

[2] S. Ankirchner and A. Dermoune, Multiperiod mean-variance portfolio optimization via market cloning. Appl. Math. Optim. 64 (2011) 135-154.

[3] A. Bensoussan, K.C.J. Sung, S.C.P. Yam, and S.P. Yung, Linear-quadratic mean field games. Preprint arXiv: 1404.5741 (2014).

[4] T. Björk and A. Murgoci, A general theory of Markovian time inconsistent stochastic control problems. Technical report, Stockholm School of Economics (2010).

[5] T. Björk, A. Murgoci and X.Y. Zhou, Mean-variance portfolio optimization with state-dependent risk aversion. Math. Finance $24(2014) 1-24$.

[6] R. Buckdahn, B. Djehiche and J. Li, A general stochastic maximum principle for SDEs of mean-field type. Appl. Math. Optim. 64 (2011) 197-216.

[7] R. Carmona and F. Delarue, Forward-backward stochastic differential equations and controlled McKean-Vlasov dynamics. Preprint arXiv: 1303.5835 (2013).

[8] R. Carmona, F. Delarue and A. Lachapelle, Control of McKean-Vlasov dynamics versus mean field games. Math. Fin. Econ. 7 (2013) 131-166.

[9] W.H. Fleming and H.M. Soner, Controlled Markov Processes and Viscosity Solutions. Vol. 25 of Stoch. Model. Appl. Probab., 2nd edition. Springer, New York (2006).

[10] D. Li and W.-L. Ng, Optimal dynamic portfolio selection: multiperiod mean-variance formulation. Math. Finance 10 (2000) $387-406$.

[11] H. Markowitz, Portfolio selection. J. Finance 7 (1952) 77-91.

[12] H.P. McKean, A class of Markov processes associated with nonlinear parabolic equations. Proc. Natl. Acad. Sci. USA 56 (1966) 1907-1911.

[13] A.-S. Sznitman, Topics in propagation of chaos. In Ecole d'Eté de Probabilités de Saint-Flour XIX - 1989. Edited by P.-L. Hennequin. Vol. 1464 of Lect. Notes Math. Springer-Verlag, Berlin (1991) 165-251.

[14] X.Y. Zhou and D. Li, Continuous-time mean-variance portfolio selection: a stochastic LQ framework. Appl. Math. Optim. 42 (2000) 19-33. 